\title{
Pre and Post Radiotherapy Imaging findings of Aggressive Vertebral Haemangioma of the Thoracicspine
}

Manjit Bagul, Rajesh Mehra

Consultant Radiologist, Venus Diagnostic Centre, Bhopal, Madhya Pradesh, 462011, India.

\section{Abstract:}

Vertebral hemangiomas $(\mathrm{VH})$ are common unifocal or multifocal benign lesions in spine imaging with incidence of $10 \%-12 \%$. Patients are usually asymptomatic and often vertebral hemangioma is incidental finding on imaging. An aggressive or atypical vertebral hemangiomas have associated imaging features like extraosseous extension, compression fractures, vertebral ballooning \& soft tissue components exist with incidence of $1 \%$ to $3 \%$. Treatment protocols for aggressive VHs include combination of radiotherapy, vertebroplasty, alcohol injection, embolization, and surgery. We present a case of 24 year female patient having aggressive D1 2 vertebral hemangioma showing extra-osseous extension, ballooning and small intra-spinal soft tissue component not causing cord compression and follow up imaging after completion of radiotherapy.

Key words: Compression Fractures, Hemangioma, Spinal Cord Compression, Spine, Vertebroplasty.

\section{Introduction}

Vertebral hemangiomas (VH), which are usually considered to be benign, are very commonly encountered lesions on radiographs, computed tomography (CT), and magnetic resonance imaging (MRI). An aggressive or atypical vertebral hemangiomas can extend outside bony margins leading to the expansion or ballooning of vertebra, compression fractures and associated soft tissue component can cause cord compression.

\section{Case Report}

A 24-year-old female patient presented with severe low back pain with no significant radiculopathy since 2-3 months. No history of fever, any chronic illness, surgery or imaging was elicited. Physical examination revealed thin built with weight: 45 $\mathrm{kg}$ and height: $141 \mathrm{~cm}$. Spine mobilization and tenderness at dorso-lumbar junction led to loss of lumbar lordosis, thoracolumbar pain pressure, contractures of the paravertebral muscles and back pain. However, patellar reflexes were normal with no neurological deficit.

Plain radiographs of the thoracolumbar spine showed grid appearance at D12 vertebra with mild ballooning and distortion of posterior vertebral wall. Pre- and post-contrast MRI of the DL

\section{Corresponding Author: Dr. Maniit Bagul}

Email: manjitbagul84@gmail.com

Received: June 25, 2016 | Accepted: June 29, 2016 | Published Online: June 30, 2016

This is an Open Access article distributed under the terms of the Creative Commons Attribution License (creativecommons.org/licenses/by/3.0)

Conflict of interest: None declared | Source of funding: Nil | DOl: http://dx.doi.org/10.17659/01.2016.0069 
spine was performed. $\mathrm{T} 1$ \& $\mathrm{T} 2 \mathrm{Wtd}$ images revealed expansile D1 2 vertebral lesion of heterogeneously hypointense T1 signal with focal hyper-intensities and heterogeneously hyperintense signal on $\mathrm{T} 2 \mathrm{Wtd}$ images [Fig.1,2]. STIR images reveals hyperintense signal with suppressed $\mathrm{T} 1$ focal hyper-intensities confirming fat signal in vertebral lesion. Ballooning \& retropulsion of posterior D1 2 vertebral wall noted compressing ventral thecal sac and encroaching over lower end of cord without significant central canal stenosis or cord compression. Extension of vertebral lesion into bilateral pedicle noted with spared bony lamina and facets. Post-contrast study shows heterogeneous enhancement in the D12 vertebral lesion with mild anterior epidural enhancing soft tissue component [Fig.3,4]. Peculiar "polka dot" appearance was seen on axial CT images and "corduroy cloth or jail bar" appearance was seen on sagittal and coronal reformatted CT images in D1 2 vertebral body [Fig.5,6].

Aggressive hemangioma of D12 vertebra of type IV (Tomita's surgical classification of spinal tumors) was diagnosed based on these characteristic findings and further investigation including angiography was advised. Patient refused for surgical intervention including the biopsy and histopathological study. She underwent radiotherapy and was followed by MR imaging after six months.

Follow-up MRI after six months, revealed no significant change in findings at the D1 2 vertebral level with unchanged intraosseous lesion and associated small intra-spinal anterior epidural soft tissue component. No signs of cord compression noted with no significant central canal stenosis at same level. No evidence of D1 2 vertebral collapse with persistent ballooning, retropulsion and distortion of posterior vertebral wall. Additional signal changes in the adjacent D11 and L1 vertebrae were noted with fatty marrow infiltration on $\mathrm{T} 1$ imaging
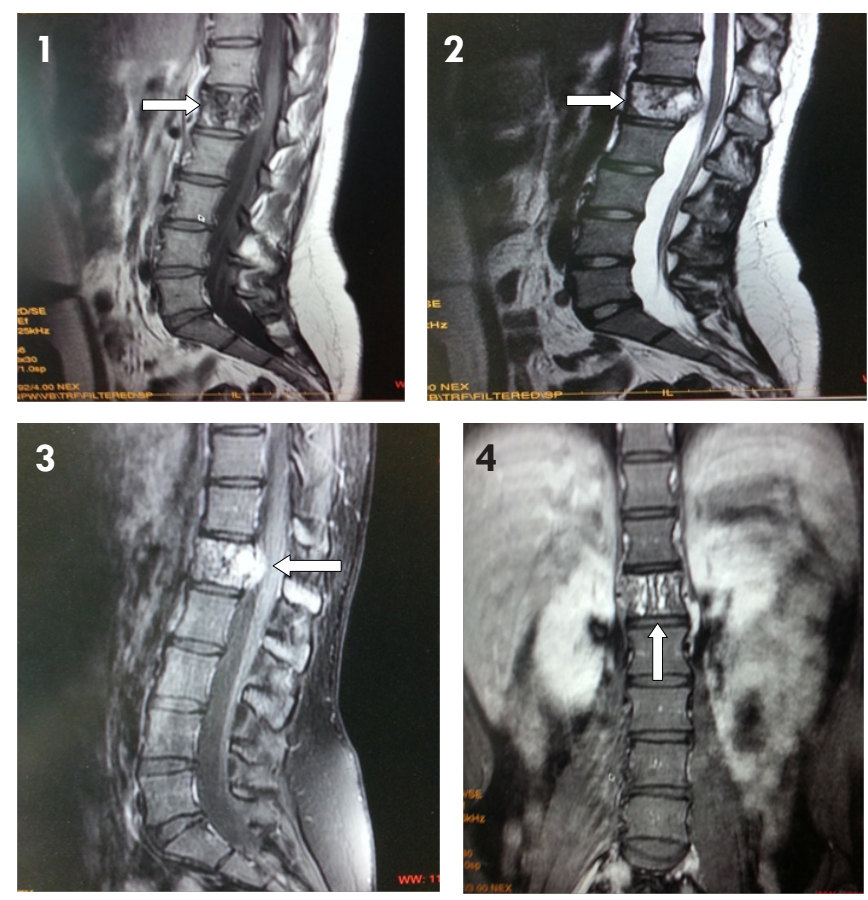

Fig.1-4: Pre-radiotherapy MRI Images SAG T1, SAGT2 \& post-contrast T1 Wtd Images showing D1 2 aggressive vertebral hemangioma with contrast enhancing intraspinal anterior epidural soft tissue compressing thecal sac (Trasitional vertebra at LS junction with sacralized L5).
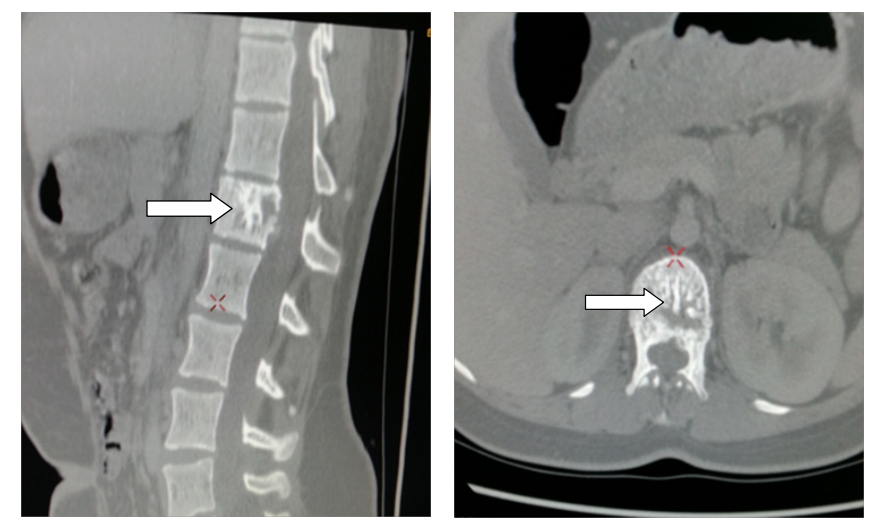

Fig.5,6: Pre-radiotherapy sagittal and axial CT images of aggressive D 12 vertebral hemangioma with corduroy cloth like appearance. 
consistent to that of post-radiation marrow changes [Fig.7].

\section{Discussion}

Vertebral hemangioma, common benign lesion of spine was first described by Perman, in 1926, followed by Bailey and Bucy in 1930. Although typically incidental findings in 10\%-12\% cases, they are symptomatic in 0.9 to $1.2 \%$ of adults $[1,2]$. Symptomatic or aggressive hemangiomas are characterized by bone expansion, extra osseous extension, compression fractures, soft tissue component \& neurological complication [1]. Approximately $45 \%$ of aggressive hemangiomas are associated with neurologic deficits, the others only characterized by pain [3]. Our patient presented with backache without any neurological deficits. They are often found incidentally by plain radiographs, CT and/or MRI $[5,6]$.

Vertebral hemangiomas are characterised by sparing and thickening of vertically striated trabeculae which preserve the functional capability of the vertebral body to withstand an axial load. This appearance has been described on radiographs and CT as "polka-dot", "honeycomb", "corduroy cloth", "jail bar", and "salt and pepper", with decrease in the overall density of the vertebral body due to the presence of fatty marrow [4]. In our case, polka dot and corduroy cloth or jail bar appearance was seen on axial CT images in D12 vertebral body. Intralesional fat of hemangiomas causes increased signal intensity on $\mathrm{Tl}$ weighted MR images. On T2-weighted images, the signal intensity increases due to high water content [7]. T2-hyperintensity is often greater than that of fat, thereby differentiating hemangiomas from focal fat deposition [8].

Aggressive hemangiomas characteristically have reduced fat content and more vascular component causing hypointensity on $\mathrm{Tl}$-weighted

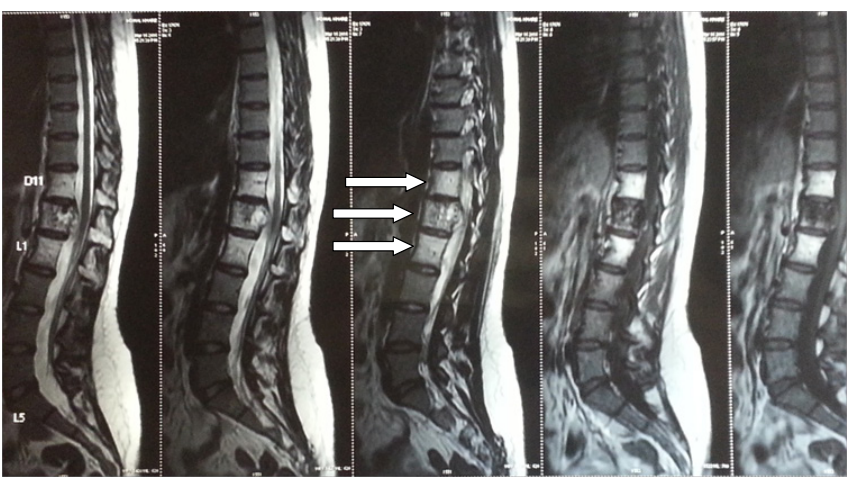

Fig.7: Post-radiotherapy MRI images SAGT1 and SAGT2 showing D1 2 aggressive vertebral hemangioma with intraspinal anterior epidural soft tissue compressing thecal sac and postradiotherapy changes in the D1 1 \& 12 vertebral bodies.

MRI $[9,10]$. Mixed signal on T1 Wtd images compared to hypointense signal and presence of coarse trabeculae help to differentiate between vertebral metastasis and aggressive haemangioma [8]. CT is helpful to accurately depict the osseous remodelling and typical pattern of vertebral haemangioma [1 1]. Paget's disease of the spine can have similar appearance but is usually distinguished by expansion of the vertebral body with peripheral cortical thickening which can be observed on CT, with characteristic "picture frame" vertebral body on radiographs [12]. Lymphoma may also have a similar appearance but is usually distinguished by homogeneous enhancement on contrast enhanced MRI [13]. Aggressive vertebral hemangiomas most often occur in thoracic spine and occupy the entire vertebral body, extend into the neural arch, expand the osseous margins, and contain a soft tissue component [14]. Cord compression and subsequent myelopathy may result from either encroachment of extradural soft tissue, pathologic fracture or haemorrhage [15].

Our patient presented with type IV aggressive symptomatic vertebral hemangioma 
as per Tomita's surgical classification of spinal tumours [16]. Reported treatment protocol for type IV includes radiotherapy, vertebroplasty, direct alcohol injection, embolization arteries, surgery, and a combination of these modalities. Successful results have been reported for each of these modalities. Our patient refused for the surgical treatment option \& opted for radiotherapy. Follow up MR imaging after completion of radiotherapy reveals no interval change in the morphology of aggressive D12 $\mathrm{VH}$ with persistent epidural soft tissue component not causing cord compression or canal stenosis. Disease was stable with no significant regression or progression.

\section{Conclusion}

Vertebral hemangiomas are usually asymptomatic benign lesions with good prognosis however symptomatic and aggressive cases of vertebral hemangiomas can cause rapid onset of neurological deficits. Imaging plays important role in the diagnosis and management of $\mathrm{VH}$. Multidisciplinary approach is needed to treat the aggressive symptomatic $\mathrm{VH}$ as in our case. Radiotherapy alone failed to change the course of disease.

\section{References}

1. Chen HI, Hever GG, Zaghloul K, Simon SL, Weigele JB, Grady MS. Case report. Lumbar vertebral hamangioma presenting with the acute onset of neurological symptoms. J Neurosurg Spine. 2007;7:80-85.

2. Cheung NK, Doorenbosch X, Christie JG. Rapid onset aggressive vertebral haemangioma. Childs Nerv Syst. 2011 ;27:469-472.

3. Pastushyn A, Slin'ko El, Mirzoyeva GM. Vertebral hemangiomas: diagnosis, management, natural history and clinicopathological correlates in 86 patients. Surg Neurol. 1998;50:535-547.

4. Grossman RI, Yousem DY. Nondegenerative diseases of the spine. In: Neuroradiology, $3^{\text {rd }}$ edn. Philadelphia; Mosby; 2003:827-828.
5. Alexander J, Meir A, Vrodos N, Yau YH. Vertebral hemangioma an important differential in the evaluation of locally aggressive spinal lesions. Spine. 2010;35(18):E920-E923.

6. Acosta FL Jr, Sanai N, Chi JH, Dowd CF, Chin $C$, Tihan $T$, et al. Comprehensive management of symptomatic and aggressive vertebral hemangiomas. Neurosurg Clin $\mathrm{N} A m$. 2008; 19(1):17-29.

7. Ross JS, Masaryk TJ, Modic MT, Carter JR, Mapstone T, Dengel FH. Vertebral hemangiomas: MR imaging. Radiology. 1987;165:165-169.

8. Quinn S. Vertebral Hemangioma. MRI Web Clinic. 2006. Available at: https.//www.radsource.us Accessed 2 March, 2011.

9. Laredo JD, Assouline E, Gelbert F, Wybier M, Merland JJ, Tubiana JM. Vertebral hemangiomas: fat content as a sign of aggressiveness. Radiology 1990;177:467-472.

10. Fox MW, Onofrio BM. The natural history and management of symptomatic and asymptomatic vertebral hemangiomas. J Neurosurg. 1993;78:36-45.

11. Cross JJ, Antoun NM, Laing RJ, Xuereb J. Imaging of compressive vertebral haemangiomas. Eur Radiol. 2000;10:997-1002.

12. Smith SE, Murphey MD, Motamedi K, Mulligan ME, Resnik CS, Gannon FG. From the Archives of the AFIP: Radiologic Spectrum of Paget Disease of Bone and Its Complications with Pathologic Correlation. Radiographics. 2002;22:11911216.

13. Boukobza M, Mazel C, Touboul E. Primary vertebral and spinal epidural non-Hodgkin's lymphoma with spinal cord compression. Neuroradiology. 1996;38(4):333-337.

14. Laredo JD, Reizine D, Bard M, Merland JJ. Vertebral hemangiomas: radiologic evaluation. Radiology. 1986;161:183-189.

15. Dickerman RD, Bennett MT. Acute spinal cord compression caused by vertebral hemangioma. Spine J. 2005;5:582-584; author reply 584.

16. Tomita K, Kawahara N, Baba H, Tsuchiya H, Fujita T, Toribatake Y. Total En Bloc Spondylectomy: A New Surgical Technique for Primary Malignant Vertebral Tumors. Spine. 1997;22:324-333. 